\title{
p53-dependent elimination of aneuploid mitotic offspring by entosis
}

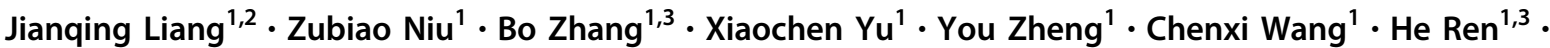 \\ Manna Wang ${ }^{1,4}$ - Banzhan Ruan ${ }^{1} \cdot$ Hongquan Qin $^{1,4} \cdot$ Xin Zhang $^{1,5} \cdot$ Songzhi Gu ${ }^{1}$ Xiaoyong Sai ${ }^{6}$ - Yanhong Tai ${ }^{7}$.

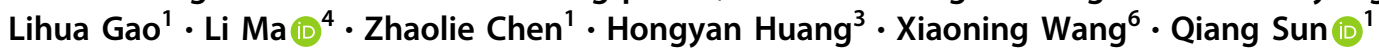

Received: 22 April 2020 / Accepted: 12 October 2020 / Published online: 27 October 2020

(c) The Author(s), under exclusive licence to ADMC Associazione Differenziamento e Morte Cellulare 2020

\begin{abstract}
Entosis was proposed to promote aneuploidy and genome instability by cell-in-cell mediated engulfment in tumor cells. We reported here, in epithelial cells, that entosis coupled with mitotic arrest functions to counteract genome instability by targeting aneuploid mitotic progenies for engulfment and elimination. We found that the formation of cell-in-cell structures associated with prolonged mitosis, which was sufficient to induce entosis. This process was controlled by the tumor suppressor p53 (wild-type) that upregulates Rnd3 expression in response to DNA damages associated with prolonged metaphase. Rnd3-compartmentalized RhoA activities accumulated during prolonged metaphase to drive cell-in-cell formation. Remarkably, this prolonged mitosis-induced entosis selectively targets non-diploid progenies for internalization, blockade of which increased aneuploidy. Thus, our work uncovered a heretofore unrecognized mechanism of mitotic surveillance for entosis, which eliminates newly born abnormal daughter cells in a p53-dependent way, implicating in the maintenance of genome integrity.
\end{abstract}

\section{Introduction}

Cell division via mitosis is strictly regulated to ensure the production of healthy daughter cells. During mitosis, correct segregation and distribution of genetic materials

These authors contributed equally: Jianqing Liang, Zubiao Niu, Bo Zhang, Xiaochen $\mathrm{Yu}$

Edited by G. Melino

Supplementary information The online version of this article (https:// doi.org/10.1038/s41418-020-00645-3) contains supplementary material, which is available to authorized users.

Hongyan Huang

hhongy1999@126.com

$\triangle$ Xiaoning Wang

xnwang88@163.com

$\triangle$ Qiang Sun

sunq@bmi.ac.cn

1 Laboratory of Cell Engineering, Institute of Biotechnology, 20 Dongda Street, Beijing 100071, China

2 State Key Laboratory of Genetic Engineering, School of Life Science, Fudan University, 2005 Songhu Road, Shanghai 200438, China into daughter cells rely on proper activation of spindle assemble checkpoint (SAC) [1,2]. SAC is activated by unsatisfied microtubule-kinetochore attachment, leading to the recruitment of SAC proteins MAD2, BUBR1, and BUB3 together with CDC20 [3] to kinetochores to form mitotic checkpoint complex (MCC). MCC functions to inhibit anaphase-promoting complex or cyclosome (APC/ $\mathrm{C})$, the multisubunit E3 ubiquitin ligase essential for metaphase-anaphase transition in the presence of CDC20. Molecules disrupting MCC such as CUEDC2 could activate $\mathrm{APC} / \mathrm{C}$ via preventing MAD2 from binding to CDC20 [4]. Activated APC/C promotes proteasomedependent degradation of cyclin B1 (CCNB1) to allow mitotic exit, and securin to release separase (ESPL1)

3 Department of Oncology, Beijing Shijitan Hospital of Capital Medical University, 10 TIEYI Road, Beijing 100038, China

4 Institute of Molecular Immunology, Southern Medical University, Guangzhou 510515, China

5 Department of Pediatric Hematology and Oncology, Xinhua Hospital affiliated to Shanghai Jiao Tong University School of Medicine, Shanghai 200092, China

6 National Clinic Center of Geriatric \& the State Key Laboratory of Kidney, the Chinese PLA General Hospital, Beijing 100853, China

7 The 307 Hospital, 8 Dongda Street, Beijing 100071, China 
which leads to cohesion destruction and subsequent sister chromatid separation [1, 2]. Prolonged SAC activation due to mitotic aberrations generally leads to mitotic arrest and often, but not always, to mitotic catastrophe (specifically referred to catastrophic death here) [5]. Those that eventually pass mitotic arrest are prone to produce aneuploid/polyploid (non-diploid) daughter cells that need to be dealt with post mitotically, otherwise impair tissue homeostasis and/or contribute to tumorigenesis $[6,7]$.

Entosis is a recently defined non-apoptotic cell death program, where suspended epithelial cells actively penetrate into and die inside of their neighbors [8]. Cell penetration leads to the formation of so called "cell-in-cell" structures (CIC), which requires polarized actomyosin contraction at the rear cortex of the internalizing cells. The polarized distribution of actomyosin is established by local inhibition of RhoA-ROCK signaling at cell-cell junctions, where lie junction-associated inhibitors, such as p190A RhoGAP [9]. A vinculin-enriched structure, named mechanical ring, interfaces between contractile actomyosin and cell-cell junction to coordinate CIC formation [10]. Multiple factors, including CDKN2A [11], PCDH7 [12], IL-8 [13] and membrane cholesterol and lipids [14], that affects RhoA signaling were recently identified as important regulators of entotic CIC formation. Although initially viable, majority of the internalized cells die non-autonomously with the assistance of outer host cells [15]. Pathologically in the context of tumors, entosis was proposed as a cellular mechanism of cell competition that selects winner tumor cell clones via internalizing and killing loser cells [16, 17], and promotes tumor evolution by inducing genome instability of outer cells $[18,19]$. However, its roles in physiological context remain largely speculative and mysterious up to date.

The tumor suppressor p53 was known as "guardian of genome". In responding to acute DNA damages, signaling cascades involving ATM/ATR and/or CHK1/CHK2 were initiated to activate p53, which was believed to trigger cellular responses such as cell cycle arrest or apoptosis to counteract the genotoxic stresses [20]. p53 initiates different cellular responses generally via regulating expression of specific downstream target genes, such as the CDK inhibitor p21 for cell cycle arrest and the pro-apoptotic Bcl-2 family members Puma and Noxa for apoptosis [21]. While cell cycle arrest was envisaged to provide cells an opportunity to fix the repairable DNA damages before next round of cell cycle and thus prevent propagation of potentially harmful mutations, cell death by apoptosis was likely a more aggressive and efficient way to eliminate cells harboring more severe genetic aberrations, and thereby to maintain genome integrity [21, 22]. In addition to apoptosis, p53 was also implicated in other forms of cell deaths that play roles in removing cells with DNA damages [22]. Mitotic catastrophe was believed to be a p53-regulated non-apoptotic cell death that eliminates questionable cells during prolonged mitosis which generally takes place with DNA damages [23]. Nevertheless, it is unknown whether genetically questionable progenies following mitosis were actively removed by a non-apoptotic cell death that might also be regulated by $\mathrm{p} 53$.

Here, we demonstrate that p53 mediates the internalization and elimination of aneuploidy daughter cells from transient mitotic arrest by entosis, which was driven by Rnd3-compartmentalized RhoA activities in daughter cells that were internalized in response to DNA damages during prolonged metaphase. Thus, our work identified a novel surveillance mechanism for entosis, in a wild-type p53dependent way, to safeguard mitosis and genome integrity.

\section{Materials and methods}

\section{Cell culture and constructs}

MCF7 and 293FT cells were maintained in Dulbecco's modified Eagle's medium (DMEM) supplemented with $10 \%$ fetal bovine serum (PAN-Biotech). MCF10A and its derivatives were cultured in DMEM/F12 supplemented with 5\% horse serum (GIBCO, \#16050-122), $20 \mathrm{ng} / \mathrm{ml}$ EGF (Peprotech, \#96-AF-100-15-100), $10 \mu \mathrm{g} / \mathrm{ml}$ insulin (Sigma, I-5500), $0.5 \mu \mathrm{g} / \mathrm{ml}$ hydrocortisone (Macgene, CC103), and $100 \mathrm{ng} / \mathrm{ml}$ cholera toxin (Sigma, C8052). HMEC cells, a gift from Dr. Qinong Ye, were cultured in Cascade Biological $^{\mathrm{TM}}$ Medium 171 (Gibico, M-171-500) supplemented with Mammary Epithelial Growth Supplement (Macgene, cc022) containing Bovine Pituitary Extract (0.4\%), EGF (3 $\mathrm{ng} / \mathrm{ml})$, hydrocortisone $(0.5 \mu \mathrm{g} / \mathrm{ml})$ and insulin $(5 \mu \mathrm{g} / \mathrm{ml})$. pBabe-H2B-mCherry was a gift from Dr. Michael Overholtzer. pBabe-RhoA biosensor was a gift of Dr. Klaus Hahn from Addgene (12602). pLKO-shp53 was a gift of Dr. Bob Weinberg from Addgene (19119). GFP-p53 was a gift of Dr. Tyler Jacks from Addgene (12091). Expression plasmid for GFP-Rnd3 was constructed by inserting synthesized human Rnd3 ORF into pQCXIP-GFP vector by Xho I and Bam H I sties. Expression plasmid for MLCmCherry was constructed by inserting synthesized chicken MLC ORF into pBabe-mCherry vector by Xho I and BamH I sties.

\section{Antibodies and chemical reagents}

Antibodies with working dilution factors, company source, and catalog number include: anti-pMLC (1:200; Cell Signaling; \#3671), anti-E-cadherin (1:200; BD Biosciences; 610181), anti- $\gamma \mathrm{H} 2 \mathrm{AX}$ (1:200; Cell Signaling; \#9718 for IF; 1:500; ABclonal; AP0099 for WB), anti-H2AX (1:1000; ABclonal; AP0823), anti-pATM (1:500; Boster; BM4008), 
anti-ATM (1:1000; Proteintech; 27156-1-AP), anti-pp53 (1:500; Cell Signaling; \#9286), anti-p53 (1:1000; Santa Cruz; sc-126), anti-Rnd3 (1:1000; Sino Biological; 101056T32), anti-cleaved caspase3 (1:1000; Cell Signaling; \#9664). Secondary antibodies include Alexa Fluor 568 anti-mouse (1:500; Invitrogen; A11031), Alexa Fluor 568 anti-rabbit (1:500; Invitrogen; A11036), Alexa Fluor 488 anti-mouse (1:500; Invitrogen; A11029), Alexa Fluor 488 anti-rabbit (1:500; Invitrogen; A11034), Alexa Fluor ${ }^{\circledR} 647$ Phalloidin (1:200; Invitrogen; A22287). DAPI was purchased from Sigma (D8417). ROCK inhibitor Y27632 was purchased from TOCRIS (1254) and used at final concentration of $10 \mu \mathrm{M}$. Ro-3306 (HY-12529), a potent and selective inhibitor of CDK1, and SU-9516 (HY-18629), a selective CDK2 inhibitor, were purchased from MedChem Express. DNA damage inducers Mitomycin (T6890) and Bleomycin (T6116), and inhibitors for ATM (T2474), ATR (T3338), CHK1 (T2033), CHK2 (T7080) were purchased from TargetMol. Z-VAD-FMK (s7023) was purchased from Selleck. Collagen Type I is a product of BD Biosciences (\#354236).

\section{Virus production and infection}

Stable expression cell lines were established by virus infection. Briefly, $1 \times 10^{6} 293 \mathrm{FT}$ cells were plated into a six-well plate coated with collagen I (BD Bioscience, \#354236), transfection was performed with retroviral constructs together with packaging plasmids, and viruses were collected twice at $24 \mathrm{~h}$ intervals. To infect cells, cells were cultured in $1 \mathrm{ml}$ viral supernatant mixed with $1 \mu \mathrm{l}$ polybrene of $10 \mu \mathrm{g} / \mathrm{ml}$ stock for $6 \mathrm{~h}$ followed with regular media. Cells were selected with appropriate antibiotics $(2 \mu \mathrm{g} / \mathrm{ml}$ puromycin or $400 \mu \mathrm{g} / \mathrm{ml} \mathrm{G} 418$ for MCF10A, $1 \mu \mathrm{g} / \mathrm{ml}$ puromycin for MCF7) for 7 days.

\section{RNA interference}

siRNAs were from GenPharma (Shanghai, China). For individual siRNA transfection, cells $\left(1 \times 10^{5} /\right.$ well $)$ were plated into a 12-well glass bottom plate and cultured overnight, then transfected with $50 \mathrm{nM}$ siRNA using Lipofectamine ${ }^{\circ}$ RAiMAX (Invitrogen, \#13778-150). Cells were fed with fresh full media $6 \mathrm{~h}$ later. siRNA sequences: CUEDC2: 5'-CAUCAGA GGAGAACUUCGA-3'; CDC20: 5'-CCACCAUGAUGUU CGGGUATT-3'; MAD2: 5'-GGAAGAGUCGGGACCACA GTT-3'; BuBR1: 5'-CGGGCAUUUGAAUAUGAAATT-3'; ESPL1: 5'-GCUUGUGAUGCCAUCCUGATT-3'; H2AX: 5'-GGGACGAAGCACUUGGUAACA-3'; p53: 5'-AAGAC UCCAGUGGUAAUCUAC-3'; Rnd3-1: 5'-GAUCCUAAU CAGAACGUGAAA-3'; Rnd3-2: 5'-AUCCUAAUCAGAA CGUGAAAU-3'; ATM: 5'-GCCUCCAAUUCUUCACAGU AA-3'; ATR: 5'-GAUGAACACAUGGGAUAUUUA-3';
CHK1-1: 5'-GUGACAGCUGUCAGGAGUAUU-3'; CH K1-2: 5'-GCCCACAUGUCCUGAUCAUAU-3'; CHK2-1: 5'-GAACAGAUAAAUACCGAACAU-3'; CHK2-2: 5'-CG CCGUCCUUUGAAUAACAAU-3'; 53BP1: 5'-GAGAGCA GAUGAUCCUUUA-3'; USP28-1: 5'-CUGCAUUCACCUU AUCAUU-3'; Negative Control: 5'-UCUCCGAACGUGUC ACGUTT- $3^{\prime}$.

\section{Reverse transcription-quantitative PCR}

Total RNA was isolated from cells $48 \mathrm{~h}$ after siRNA transfection using TRIzol reagent (Invitrogen, \#15596026). One microgram of total RNA was converted into cDNA using TransScript $t^{\oplus}$ One-Step gDNA Removal and cDNA Synthesis SuperMix (Transgen Biotech, \#AT311-02) according to manufacturer's instruction. The quantitative PCR (qPCR) was performed on $15 \mathrm{ng}$ of cDNA from each sample using SYBR Green Real-time PCR Master Mix (TOYOBO, \#QPK-201) based on the recommendations of manufacturer. Primers pairs spanning at least two exons were confirmed by NCBI Primer-BLAST: CUEDC2: $5^{\prime}$-TG AGCGATGCCAGGAACAA- $3^{\prime}$ and $5^{\prime}$-CTCCTCCTCAGC GCCAGTT-3'; CDC20: 5'-TTCCCTGCCAGACCGTATC C-3' and 5'-CAGCCAAGTAGTTGCCCTC-3'; MAD2: $5^{\prime}$-TTCTCATTCGGCATCAACA- $3^{\prime}$ and $5^{\prime}$-TCTTTCCAG GACCTCACCA-3'; BuBR1: 5'-TCTTCAGCAGCAG AAACGG-3' and $5^{\prime}$-TCATTGCATAAACGCCCTA-3'; ESPL1: $5^{\prime}$-CCCCACTTCGGGCATTGTA- $3^{\prime}$ and $5^{\prime}$-GGG CAAAGTCATAAACCACC-3'; BUB1: 5'-AGAAATACC ACAATGACCCAA- $3^{\prime}$ and $5^{\prime}$-AGGCGTGTCTGAAATA ACC-3'; H2AX: 5'-CCCTTCCAGCAAACTCAACTCG-3' and 5'- AAACTCCCCAATGCCTAAGGT-3'; p53: 5'-AC CACCATCCACTACAACTACAT- $3^{\prime}$ and $5^{\prime}$-CTCCCAGG ACAGGCACAAA-3'; Rnd3: 5'- TCTTACCCTGATTC GGATGC- $3^{\prime}$ and $5^{\prime}$-TCTGACGCTATTTTCCGACT-3'; ATM: 5'-GCACAGAAGTGCCTCCAATTC- $3^{\prime}$ and $5^{\prime}-\mathrm{AC}$ ATTCTGGCACGCTTTG-3'; ATR: 5'-GCCGTTCTCCA GGAATACAG-3' and 5'-GAGCAACCGAGCTTGAGAG T-3'; CHK1: 5'- GGATGCGGACAAATCTTACCA-3' and 5'-CCTTAGAAAGTCGGAAGTCAACC-3'; CHK2: 5'-G TCATCTCAAGAAGAGGACT- $3^{\prime}$ and $5^{\prime}$ - GAGCTGTGG ATTCATTTTCC-3'; 53BP1: 5'-CCTCCACATGGCCATG TCTTAC- $3^{\prime}$ and $5^{\prime}$-CCTGAGGAGCCCCCAGTCTGT-3'; USP28-1: $5^{\prime}$-GGAACAGCAGCAAGATGTGA- $3^{\prime}$ and 5'-GGCCGAAGGTCTCATTGTTA-3'; HPRT: 5'-AGGC CATCACATTGTAGCCCTCTGT- $3^{\prime}$ and $5^{\prime}$-TACTGCCT GACCAAGGAAAGCAAAGT- $3^{\prime}$. The PCR reactions run on the following conditions: initial denaturing at $95^{\circ} \mathrm{C}$ for $30 \mathrm{~s}$, followed by $35-40$ cycles of $95^{\circ} \mathrm{C}$ for $5 \mathrm{~s}, 60^{\circ} \mathrm{C}$ for $10 \mathrm{~s}$ and $72{ }^{\circ} \mathrm{C}$ for $15 \mathrm{~s}$, melting curves were examined at $37{ }^{\circ} \mathrm{C}$ for $30 \mathrm{~s}$ before cooling. Each result was from three independent biological replicates for all analyses performed in this work. The qPCR results were analyzed using $2^{-\Delta \Delta C T}$ 
method and presented as relative quantity of transcripts with HPRT as the reference gene.

\section{Cytospin and entotic CIC quantification}

Described protocol [24] was slightly modified to examine cells abilities to form CIC structures. Briefly, cells were cultured in suspension for $6 \mathrm{~h}$ in a six-well plate pre-coated with $1 \mathrm{ml}$ solidified soft agar $(0.5 \%)$ and then mounted onto glass slides for 3-min centrifugation at $400 \mathrm{rpm}$ to make cytospin. Cells were fixed by $4 \%$ PFA and immunostained with E-cadherin antibodies followed by mounting with Antifade reagent with DAPI. CIC structures with more than half of cell body internalized were counted.

\section{Time lapse imaging and entotic CIC quantification}

Wide field imaging was performed on cells plated in glass bottom dish or plate (Nest Biotechnology Co.) by Nikon Ti-E microscope equipped with motorized stage and Neo Vacuum cooled Scientific CMOS Camera (Andor Technology). Images were collected every 10 or $15 \mathrm{~min}$ for $24 \mathrm{~h}$ using $\times 10$ or $\times 20$ Apo objective lens with $15 \mathrm{~ms}$ exposure for DIC channel and $150 \mathrm{~ms}$ exposure for mCherry channel. Cells were cultured in humidified chamber supplied with $5 \% \mathrm{CO}_{2}$ at $37^{\circ} \mathrm{C}$ during imaging. Image sequences were reviewed using Nikon NIS-Elements AR 4.5 software. Mitotic entry was judged morphologically by either condensed chromatin as indicated by H2B-mCherry condensation or cells' round up. Mitotic anaphase was judged by chromosome separation labeled with H2B-mCherry. The duration of metaphase was determined from the first frame of mitotic entry to the first frame of mitotic anaphase. CIC structures were determined morphologically by complete enwrapping of cells into their neighbors, typically with a crescent nucleus in the outer cells. Total cells in each field were counted on mCherry-positive nuclei. CIC frequency was presented as CIC number divided by total cells in each field.

For CIC formation induced by DNA damages, MCF10A/ $\mathrm{H} 2 \mathrm{~B}$-mCherry cells in a 12 -well plate $\left(2.5 \times 10^{4} /\right.$ well $)$ were synchronized by $100 \mathrm{ng} / \mathrm{m}$ nocoldazole for $6 \mathrm{~h}$ followed by treatment of DNA damage inducers mitomycin $(0.5,1$, $2 \mu \mathrm{M})$ for $3.5 \mathrm{~h}$. Then, drugs were washed out for $20 \mathrm{~h}$ time lapse in full medium. CIC formation was quantified as did above.

\section{Time lapse-associated FISH (fluorescence in situ hybridization)}

Prior to FISH, MCF10A cells of $3 \times 10^{5}$ were first cultured in gridded glass bottom dish ( $\mu$-Dish 35 mm Grid-500, ibidi;
\#81168) for $20 \mathrm{~h}$, followed by time lapse microscopy with images collected every $10 \mathrm{~min}$ for $16 \mathrm{~h}$ by using $\times 10$ Apo objective lens in DIC channel. Then, cells were immediately fixed at room temperature for 20 min with freshly prepared solution (methanol/glacial acetic acid $=3: 1$ ) after briefly washed twice with PBS. Fixed cells were initially baked at $56{ }^{\circ} \mathrm{C}$ for $30 \mathrm{~min}$, subsequently incubated in $100 \mu \mathrm{g} / \mathrm{ml}$ RNase $(\mathrm{pH}=7.0 \pm 0.2)$ for $1 \mathrm{~h}$ and then $20 \mathrm{mg} / \mathrm{ml}$ pepsin$0.01 \mathrm{M} \mathrm{HCl}$ for $10 \mathrm{~min}$ at $37^{\circ} \mathrm{C}$. After washed twice with $2 \times \mathrm{SSC}$ at room temperature for $5 \mathrm{~min}$, samples were dehydrated in $70 \%, 85 \%$, and $100 \%$ precooled ethanol for 2 min respectively, and then air-dried. Hybridization was performed with two-probe FISH kit (F01010-00, GP Medical Technologies, Ltd) following the manual provided. Briefly, sample was denatured at $75^{\circ} \mathrm{C}$ for $10 \mathrm{~min}$ and then preceded to hybridization at $42{ }^{\circ} \mathrm{C}$ for $16 \mathrm{~h}$ in a humidified cassette. Following hybridization, sample was serially washed with $0.4 \times$ SSC containing $0.3 \% \mathrm{NP}-40(\mathrm{pH}=7.0 \pm$ 0.2 ) at $65^{\circ} \mathrm{C}$ for $3 \mathrm{~min}, 2 \times \mathrm{SSC}$ containing $0.1 \% \mathrm{NP}-40$ $(\mathrm{pH}=7.0 \pm 0.2)$ at room temperature for $1 \mathrm{~min}$ and finally $70 \%$ ethanol at room temperature for $3 \mathrm{~min}$ before counterstained with DAPI in darkness for 10-15 min and mounted. Images were taken by using Ultraview Vox spinning disc confocal system (Perkin Elmer) equipped with a Yokogawa CSU-X1 spinning disc head and EMCCD camera (Hamamatsu C9100-13) on Nikon Ti-E microscope. Analysis was performed with Volocity software (Perkin Elmer). Information on cell position, division, and CIC formation were determined based on time lapse imaging and grids on the glass bottom.

\section{Time lapse-associated immunostaining (TLAS)}

For phospho-Myosin Light Chain 2 (pMLC) staining in mitotic cells, MCF10A/H2B-mcherry cells in gridded glass bottom dish ( $\mu$-Dish $35 \mathrm{~mm}$ Grid-500) were transfected with CDC20 siRNA as described above. Time lapse imaging was performed by $\times 10$ Apo objective lens in DIC and mCherry channels next day, with images captured every $10 \mathrm{~min}$ for $4 \mathrm{~h}$ before fixing with $4 \%$ PFA and preceded to routine staining. Briefly, the fixed sample was permeabilized with $0.2 \%$ Triton-X 100/PBS for $5 \mathrm{~min}$ and blocked with 5\% BSA for $1 \mathrm{~h}$ before incubated with primary antibody at $4{ }^{\circ} \mathrm{C}$ overnight followed fluorophore-labeled secondary antibody, cells were then co-stained with phalloidin and DAPI for $20 \mathrm{~min}$ and then mounted with Prolong Gold antifade reagent (Invitrogen). Images were captured with Nikon Ti-E microscope equipped with Neo Vacuum cooled Scientific CMOS Camera (Andor Technology) and analyzed with Nikon NISElements AR 4.5 software. Mean pMLC intensity of individual cell was calculated by equation: (pMLC-background)/ area. TLAS of $\gamma \mathrm{H} 2 \mathrm{AX}$ and p53 were performed following 
the protocol for pMLC with slight modification that time lapse was performed for $20 \mathrm{~h}$ in control or CUEDC2depleted MCF10A cells.

\section{Immunostaining and Immunoblotting}

Cultured cells were stained following protocol above in TLAS. For tissue sections, samples were first deparaffinized and antigen retrieved following routine procedures and then preceded to immunostaining as did in fixed cells above. Confocal images were captured and processed by Ultraview Vox confocal system (Perkin Elmer) on Nikon Ti-E microscope. Immunoblotting was performed following standard procedures, briefly, protein samples were separated by SDS-PAGE and then transferred onto PVDF membrane, where specific antibodies were used to probe target proteins.

\section{Flow cytometry}

MCF-7 cells of $2 \times 10^{5}$ were first cultured in a 12 -well plate overnight followed by siRNA transfection. Cells were prepared as single-cell suspensions fixed with $70 \%$ ethanol $48 \mathrm{~h}$ post transfection, and then were stained by Propidium iodide (Solarbio, CA1020) after RNase treatment before loaded on a NovoCyte flow cytometer (Agilent, USA) for analysis by the software of Novoexpress V1.4.1.

\section{FRET}

Briefly, activation levels of RhoA were measured by monitoring the ratio of ECFP to Citrine-YFP FRET and ECFP intensities $[9,25]$. Images were acquired on a Nikon Ti-E inverted microscope using a Neo Vacuum cooled Scientific CMOS Camera (Andor Technology) mounted on the bottom port with a set of excitation/emission filter wheels to direct the DIC, ECFP, FRET, and Citrine-YFP signals sequentially. Images were obtained using a Nikon 20x/0.75 CFI Plan Apochromat Lambda lens and Nikon NIS-Elements AR 4.5 software. The filter sets used for ratiometric imaging were (Excitation, emission, respectively, Chroma Technology): ECFP: ET438/24, ET482/35; FRET: ET438/24, ET540/30; and Citrine-YFP: ET513/17, ET540/30. Cells were illuminated by LUMENCOR SPECTRA X Light Engine with 438/24 and 513/17 excitation filters. CFP, FRET, and DIC images were recorded with $1 \times 1$ binning. The FRET module of Nikon NISElements AR 4.5 software was used to process image sequences. The background-subtracted images from two cameras were aligned to ascertain optimal registration with subpixel accuracy. A linear rainbow pseudocolor lookup table was applied to the ratiometric images.

\section{Patient samples}

Breast cancer sections were obtained from 307 Hospital under the hospital's regulations and ethics. Sections stained with E-cadherin or HER2 were scanned by NanoZoomerSQ (Hamamatsu) digital slide scanning system. CIC structures were judged by fully enclosing of one or more cells within another cell based on membrane contour line. Patient sections were first screened based on the number of CIC structures in fields of $\times 40$ magnification, those that had less than $1 \mathrm{CIC}$ structures in more than three fields were scored as low CIC, more than $15 \mathrm{CIC}$ structures in three fields as high CIC.

\section{Statistics}

All assays were carried out in triplicate or more. Data were expressed as means with standard deviations (SD) or standard error of mean. $P$ values were calculated using two-tailed Student's $t$ test from Excel or GraphPad Prism software, and $P$ values less than 0.05 were considered statistically significant. Logistic regression analysis in Excel was used to evaluate association between factors.

\section{Results}

\section{Entosis is associated with prolonged mitosis}

In a process of tracking entotic CIC formation by time lapse microscopy in adherent MCF10A, a non-transformed human mammary epithelial cell (HMEC) line routinely used for entosis research, we unexpectedly found that majority (22/24) of CIC structures formed shortly after mitotic cell division, in spite of a few of exceptions where one adherent cell migrating into and one suspended cells sinking into their neighbors (Fig. 1a, c and Supplementary Movie S1). Once internalized, the inner cells readily underwent cell death (Fig. 1b, d and Supplementary Fig. S1, Movie S2, 3) as previously reported for entosis [8]. The result suggested that mitosis could initiate entosis in adherent monolayers as recently reported for the induced entosis, via CDC42 depletion, in 16HBE cells [26]. However, only a small portion of the mitotic events $(<0.5 \%$ or so) led to entosis in normal adherent MCF10A culture, suggesting that entotic mitosis, referring to mitosis leading to entosis, is intrinsically different from normal mitosis. Comparison analysis revealed a significantly prolonged metaphase in entotic mitosis of MCF10A cells (Fig. 1a, e and Supplementary Movie S1, 2). Similar entosis also occurred in MCF7, a breast cancer cell line that frequently undergoes entosis (Supplementary Fig. S1c, d), indicating that entosis may take place in respond to mitotic arrest. 
a

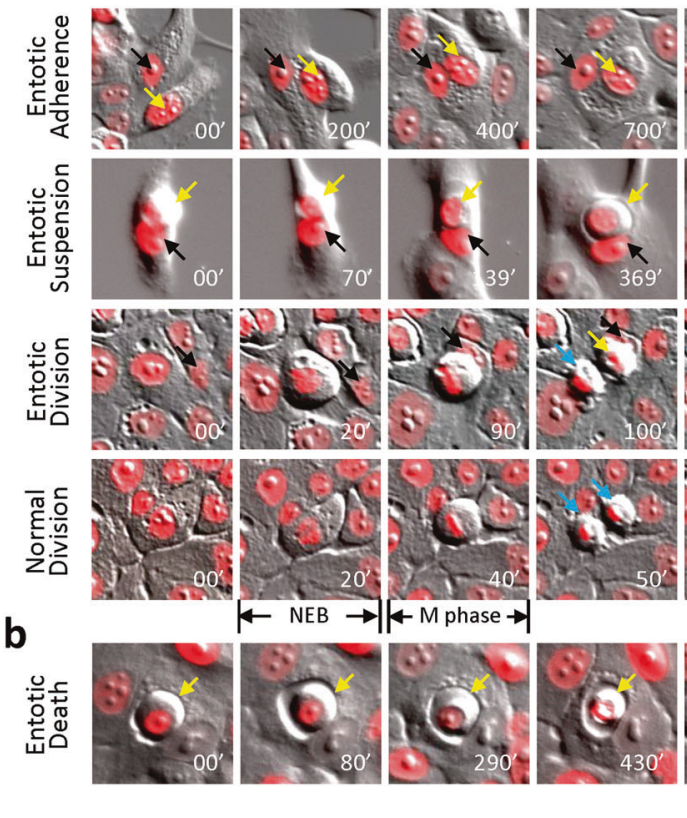

Fig. 1 Entosis is preceded by mitosis of prolonged metaphase. a Representative image sequences for the formation of entotic CIC structures from adherent cell (panel of Entotic Adherence), suspended cells (panel of Entotic Suspension), divided cells (panel of Entotic Division), and normal cell division (panel of Normal Division). Yellow arrows indicate internalized cells, black arrows indicate outer cells, blue arrows indicate cells adhered to plate bottom. NEB nuclear envelop breakdown; Scale bar: $20 \mu \mathrm{m}$. Also see Movie S1. b Representative image sequences for inner cell death of entotic CIC structures

\section{Transient mitotic arrest activates entotic CIC formation}

To test this idea, we performed RNAi-mediated knockdown (KD) of CDC20 (Fig. 2a) and ESPL1 (Supplementary Fig. S2a), two core genes critical for metaphase to anaphase transition, in MCF10A cells. Both CDC20 and ESPL1 depletion efficiently induced mitotic arrest of various extents and importantly increased CIC formation $36 \mathrm{~h}$ post siRNA transfection when a number of mitotically arrested cells started to appear (Fig. 2b, c and Supplementary Fig. S2b-d). Similar to that in spontaneous entosis, mitosis is corresponding to majority of CIC formation (47/51 for CDC20 KD, 53/54 for ESPL1 KD) for these two induced entosis (Fig. 2d and Supplementary Fig. S2e), consistent with which, blocking cell cycle with CDKs' inhibitors (Ro3306 and SU-9516) efficiently inhibited CIC formation (Fig. 2c). Interestingly, while metaphase of normal division peaks around $20 \mathrm{~min}$ for MCF10A cells, entotic mitosis displays a restricted metaphase range from 30 to $195 \mathrm{~min}$ with most entotic events preceded by mitotic arrest of $60 \mathrm{~min}$ or so (Fig. 2b,e and Supplementary Fig. S2c). Mitotic cells arrested for more than 200 min were unlikely capable of division and eventually ended up with catastrophic death (Fig. 2e, f and Supplementary Fig. S2c,
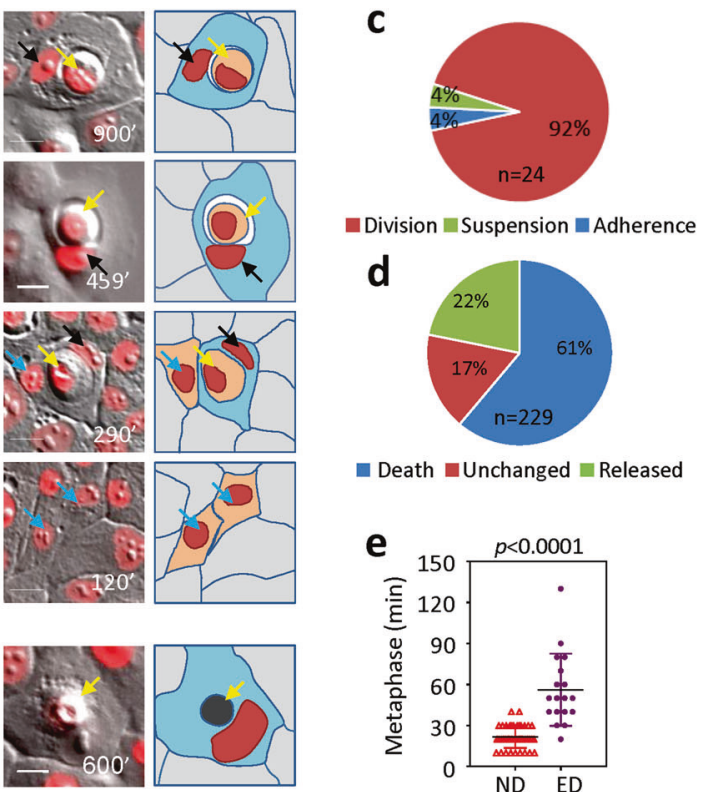

घeath nunchanged $\square$ Released

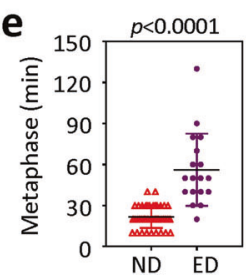

in MCF10A cells. Arrows indicate inner cell. Scale bar: $20 \mu \mathrm{m}$. c Quantification of Entotic Adherence (Adherence), Entotic suspension (Suspension), and Entotic Division (Division). Mitotic cell division (Division) plays prominent role in the formation of CIC structures in adherent MCF10A cell cultures. d Quantification of three inner cell fates in entotic CIC structures over $24 \mathrm{~h}$ period in MCF10A cells. Also see Movie S3. e Metaphase analysis of normal cell division (ND) and entotic cell division (ED) referring to cell division leading to entotic CIC formation in MCF10A cells. $n=52$ for ND, 18 for ED.

Movie S4). Therefore, mitotic catastrophe and entosis are likely cooperated to safeguard aberrant mitosis with each of them worked before and after mitosis, respectively. To further confirm the role of mitotic arrest in activating entosis, mitotic arrest induced by depleting CUEDC2, a promoter of metaphase-anaphase transition [4], was released by co-depleting either BUBR1 or MAD2 (Fig. 2g and Supplementary Fig. S2f-h), two essential components of MCC [1]. As a result, mitosis-activated CIC formation induced by CUEDC2 depletion was inhibited as well (Fig. 2h, i). Together, the data presented above support that mitotic arrest, indicated by prolonged metaphase, primes cells to undergo entotic CIC formation post mitotically.

\section{DNA damages promote entosis induced by transient mitosis arrest}

DNA damages were reported being associated with prolonged mitosis [27]. We therefore hypothesized that DNA damages might account for entosis incurred by prolonged mitosis. Consistent with this idea, TLAS indicated that cells of longer metaphase were positive in $\gamma \mathrm{H} 2 \mathrm{AX}$ (Fig. 3a, b and Supplementary Fig. S2i), a marker of DNA damages, with nuclear $\gamma \mathrm{H} 2 \mathrm{AX}$ foci significantly more than those in cells of shorter metaphase (Fig. 3c). Moreover, the nuclear $\gamma \mathrm{H} 2 \mathrm{AX}$ 

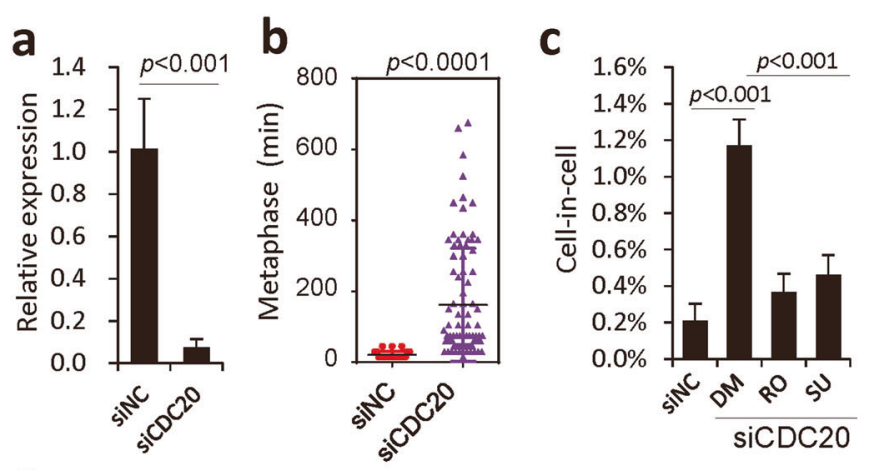

d
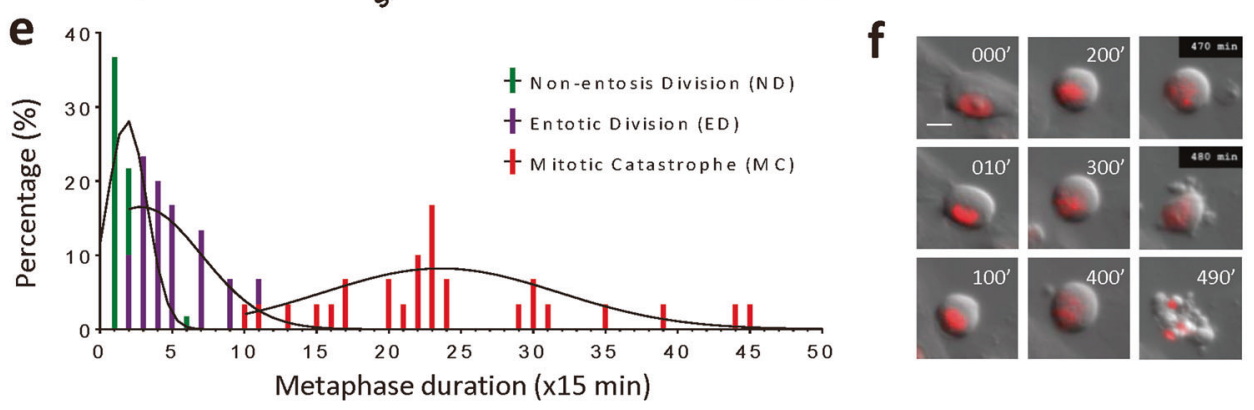

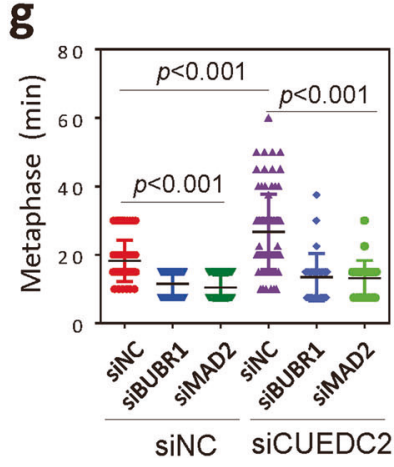

h

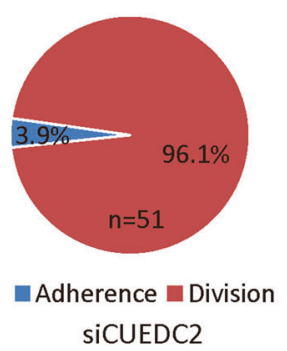

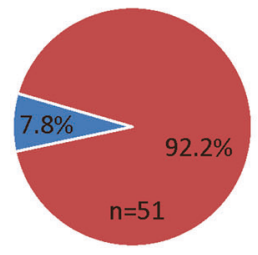

Adherence Division $\operatorname{siCDC} 20$

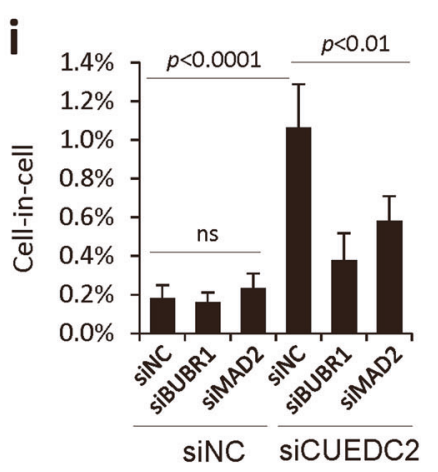

Fig. 2 Prolonged mitosis primes cells to undergo entosis. a CDC20 mRNA level examined by quantitative PCR (qPCR) upon knockdown by RNA interference. Data are mean \pm SD of triplicate experiment. $p<$ 0.001 . b Graph plots metaphase duration of control (siNC, $n=30$ ) and CDC20-depleted (siCDC20, $n=90$ ) cells. $p<0.0001$. c Quantification of CIC structures in control (siNC) and CDC20 (siCDC20) depleted cells. DM: DMSO; RO: Ro-3306(10 $\mu$ M); SU: SU-9516 (5 $\mu \mathrm{M})$. Data are mean \pm SD of 4 or more fields with more than 5000 cells analyzed each. $p<0.001$ for the pairs of siNC vs. DM, DM vs. RO, DM vs. SU. d Quantification of Entotic Adherence (Adherence) and Entotic Division (Division) in MCF10A cells with CDC20 depletion. e Histogram analysis with fitted curves of metaphase from non-entosis division (ND), entotic division (ED), and mitotic catasphrohpe (MC). $n=30$ for ND and MC, 24 for ED. Gaussian curve was created by

foci were correlated with the formation of CIC structures, where the internalizing mitotic daughter cells contained more nuclear $\gamma \mathrm{H} 2 \mathrm{AX}$ foci than their siblings that did not form CIC structures (Fig. 3d). Significantly, CIC formation was consistently suppressed by $\gamma \mathrm{H} 2 \mathrm{AX}$ depletion (Fig. 3e, f) or inhibition of its downstream signaling by chemical compounds (Fig. 3g) or siRNAs targeting ATM, ATR, CHK1, and CHK2 (Supplementary Fig. S2j, k), indicating nonlinear regression of the frequency distribution by GraphPad Prism software. f Image sequence shows example of mitotic catastrophe with 490 min metaphase. Also see Movie S4. Scale bar: $20 \mu \mathrm{m}$. g Graph plots metaphase duration of control (siNC) and CUEDC2, BUBR1, and MAD2 depleted cells. From left to right, $n=58,60,60,85,30$, 30 , respectively. $p<0.001$ between pairs analyzed as indicated. h Quantification of Entotic Adherence (Adherence) and Entotic Division (Division) in MCF10A cells with CUEDC2 depletion. i Quantification of CIC structures in cells co-depleting CUEDC2 with BUBR1 or MAD2 genes. Data are mean \pm SD of 4 or more fields with more than 5000 cells analyzed each. ns not significant. $p<0.0001$ between siCUEDC 2 and siNCs, $p<0.01$ between siNC and siBUBR 1 or siMAD2 within siCUEDC2 group.

an essential role of DNA damage signaling in entosis induced by prolonged mitosis. To directly examine the effects of DNA damages on entosis, cells synchronized in M phase were treated with mitomycin, a DNA damage inducer, and then cultured in normal media to allow mitotic exit and cytokinesis. As shown in Fig. 3h-i, mitomycin treatment efficiently activated DNA damage response (DDR), indicated by enhanced expression of $\gamma \mathrm{H} 2 \mathrm{AX}$ and 

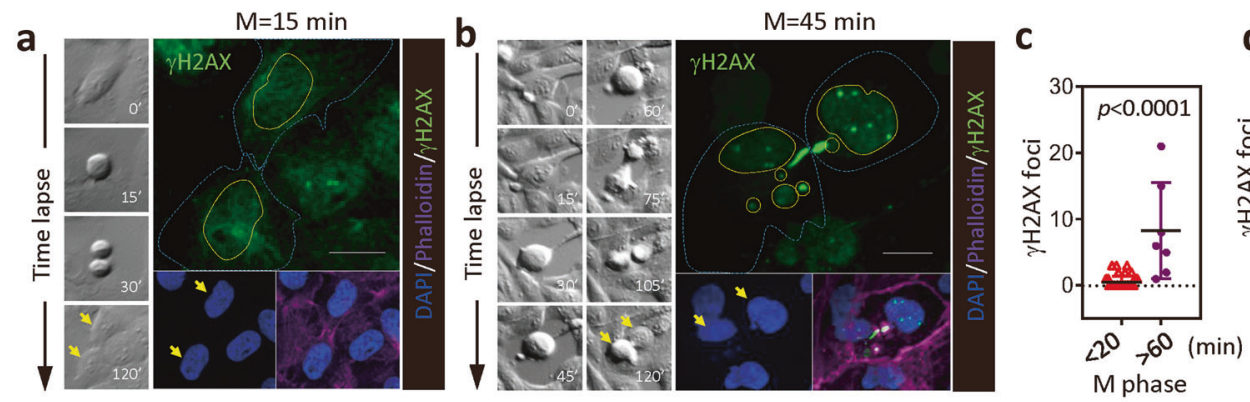

d
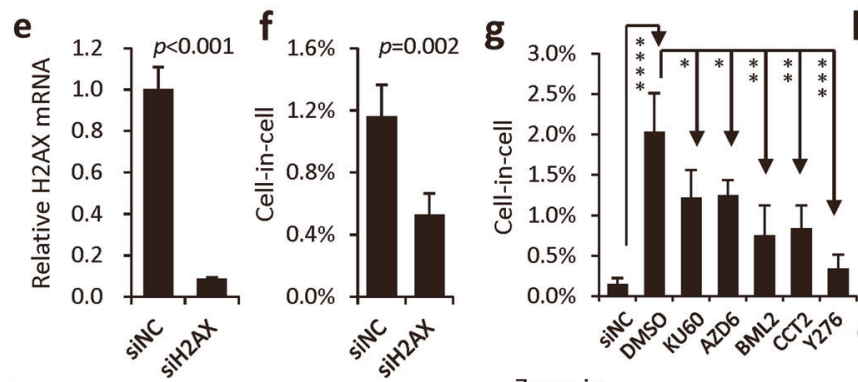

h

Noco. - + + +

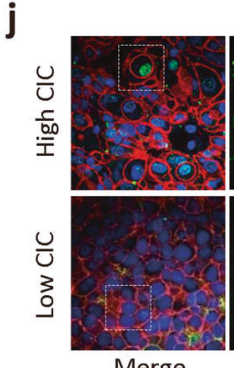

Merge

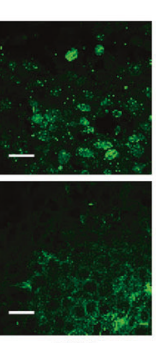

$\gamma \mathrm{H} 2 \mathrm{Ax}$

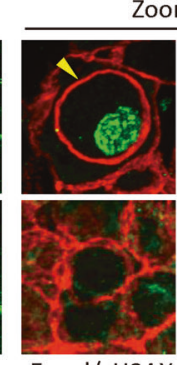

$\mathrm{E}-\mathrm{cad} / \gamma \mathrm{H} 2 \mathrm{AX}$

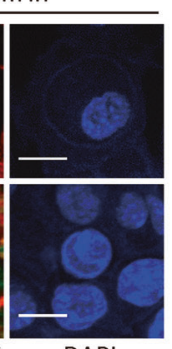

DAPI

Fig. 3 Mitotic DNA damages promote entosis. a, b Representative images for time lapse-associated immunostaining of $\gamma \mathrm{H} 2 \mathrm{AX}$ in mitotic cells of short $M$ phase (a, $15 \mathrm{~min})$ and longer $M$ phase (b, $45 \mathrm{~min})$, with corresponding DIC images of time lapse on the left and images for DAPI and merge channels on the bottom. Arrows indicate sibling cells of each division. Blue dashed lines depict cell shape, yellow dashed lines depict shape of nuclei. Note: sibling cells from longer $\mathbf{M}$ phase (b, $45 \mathrm{~min}$ ) contain micronuclei indicating chromosome missegregation, and multiple $\gamma \mathrm{H} 2 \mathrm{AX}$ foci indicating DNA damages. Scale bar: $10 \mu \mathrm{m}$. Also see Movie S5. c Number of nuclear $\gamma \mathrm{H} 2 \mathrm{AX}$ foci in cells from mitosis of different M phases. $n=48$ for M phase $<$ 20 min and 7 for $M$ phase $>60$ min. d Number of nuclear $\gamma \mathrm{H} 2 \mathrm{AX}$ foci in paired sibling cells with one attached to the culture bottom (At) while another internalized to form CIC structure (En). $n=12$. H2AX depletion (e) inhibits entotic CIC formation (f). Data are mean \pm SD of 4 or more fields with more than 5000 cells analyzed each. g Effects of inhibiting DDR signaling on entotic CIC formation in

phosphorylated ATM, and significantly increased entotic CIC formation following cell division. These effects were confirmed by bleomycin, another DNA damage inducer (data not shown). Interestingly, DNA damage signaling was likely also activated in human breast cancer tissues with high CIC structures, where nuclear $\gamma \mathrm{H} 2 \mathrm{AX}$ foci were significantly more than those in low-CIC breast cancer tissues (Fig. 3j, k). Together, these data suggest that prolonged mitosis-associated DNA damages induce

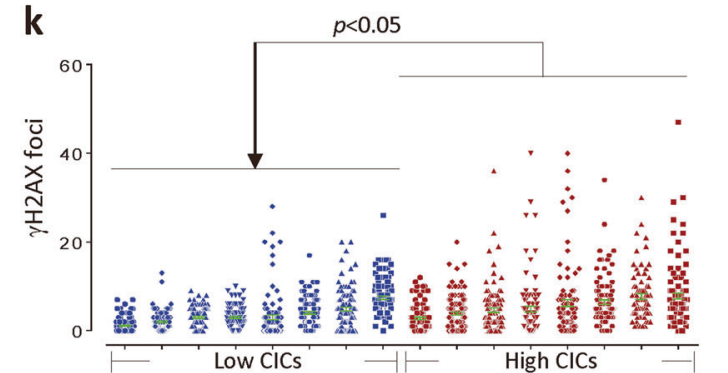

CUEDC2-depleted cells by compounds targeting ATM (KU60 for KU-60019), ATR (AZD6 for AZD6738), CHK1 (BML2 for BML277), and CHK2 (CCT2 for CCT245737). Inhibition of ROCKs by Y27632 (Y276) as positive control. Data are mean \pm SD of 4 or more fields with more than 4000 cells analyzed each. siNC for nontarget control siRNA. h DNA damages by mitomycin (Mito.) increase expression of $\gamma \mathrm{H} 2 \mathrm{AX}$ and phosphor-ATM in cells synchronized in $\mathrm{M}$ phase by nocodazole (Noco.). i Formation of entotic CIC structures in cells $18 \mathrm{~h}$ released from mitotic arrest under conditions in (h). Data are mean \pm SD of 4 or more fields with more than 4000 cells analyzed each. j Representative images for $\gamma \mathrm{H} 2 \mathrm{AX}$ staining in breast cancer tissues. E-cadherin (E-cad) in red indicates cell junctions. Arrow head indicates inner cell of a CIC structure. Scale bars: $20 \mu \mathrm{m}$ for the left, $10 \mu \mathrm{m}$ for zoomed images in the right. k Quantification of nuclear $\gamma \mathrm{H} 2 \mathrm{AX}$ foci in 16 human breast cancer samples. About one hundred of cells were quantified for each sample.

entosis. Whereas, failed cytokinesis, which is associated with prolonged mitosis, is unlikely the primary trigger of entotic CIC formation here as the lagging chromosomes/ micronuclei, a readout of failed cytokinesis, were rarely present in the mitotic daughters that were being internalized during the period of cytokinesis (Supplementary Fig. S3a, b); USP28 and 53BP1, two molecules that mediate signals from prolonged mitosis, seemed to be involved little into this process as their depletions did not 
a
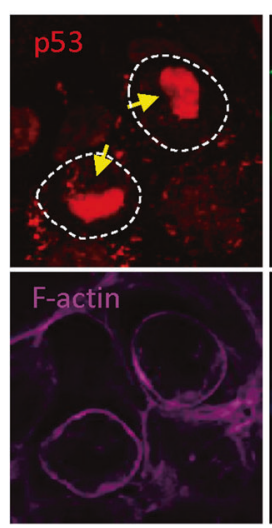

C

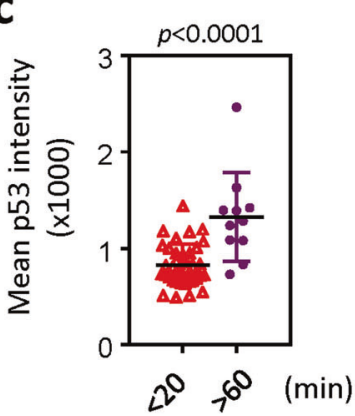

g

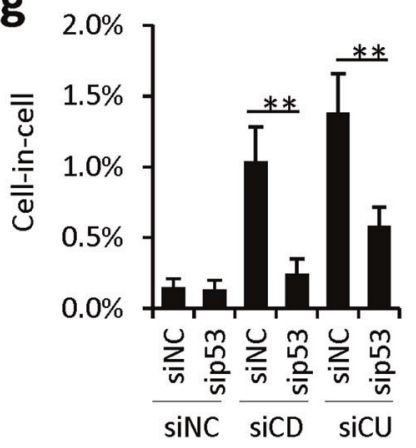

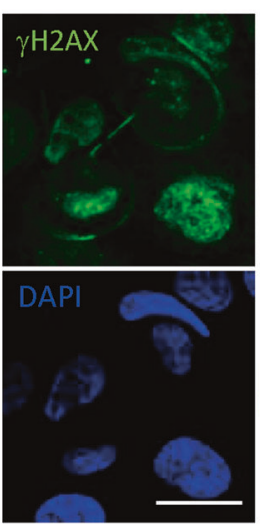
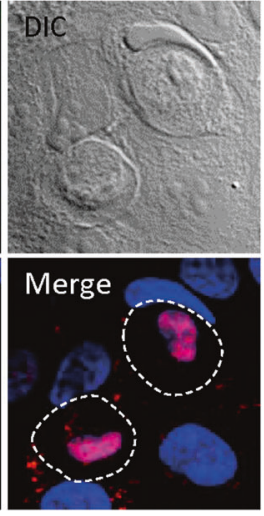

b

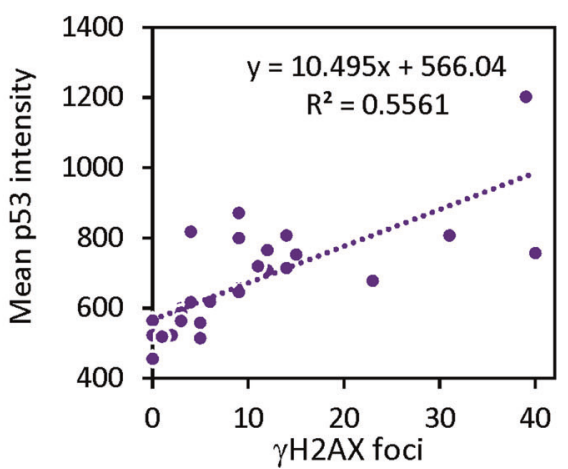

d

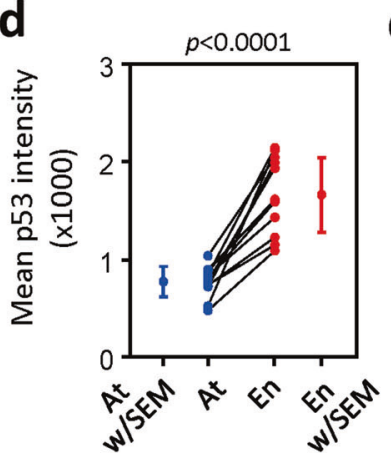

h

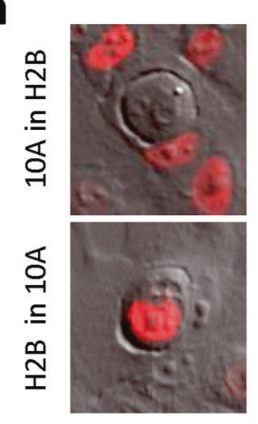

e

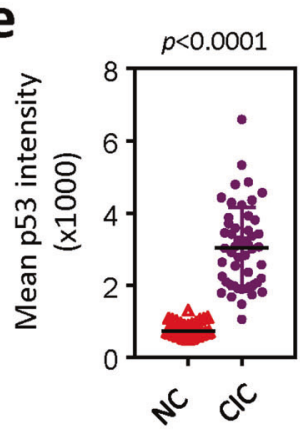

i

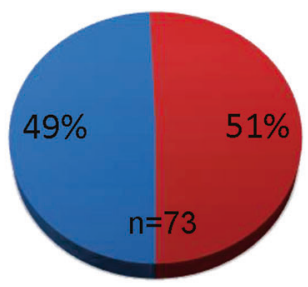

- 10A-H2B in 10A

- $10 \mathrm{~A}$ in $10 \mathrm{~A}-\mathrm{H} 2 \mathrm{~B}$

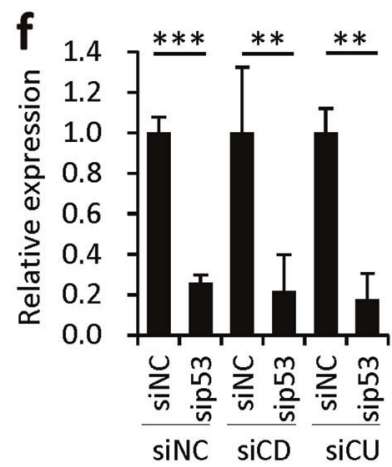

j

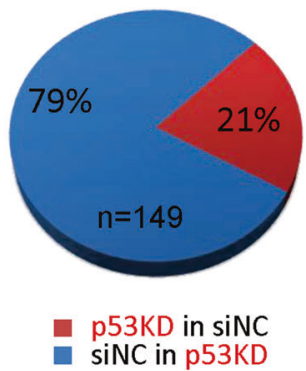

Fig. 4 p53 signaling pathway is required for entosis. a Representative images for p53 staining in daughter cells forming CIC structures as indicated by yellow arrows. Scale bar is $10 \mu \mathrm{m}$. b Correlation analysis between 553 expression and number of nuclear $\gamma \mathrm{H} 2 \mathrm{AX}$ foci in MCF10A cells. $n=30$. c Expression of p53 as indicated by mean staining intensity in cells from mitosis of different M phases. $n=38$ for M phase $<20$ min and 11 for $M$ phase $>60 \mathrm{~min}$. d Expression of p53 in paired sibling cells with one attached to the culture bottom (At) while another

significantly affect entotic CIC formation induced by CDC20- or CUEDC2-KD (Supplementary Fig. S3c-e). Also, consistent with the concept of non-apoptotic cell death program, the initiation of entosis here is apoptosisindependent as the treatment of Z-VAD, a pan-caspase inhibitor, did not inhibit CIC formation either at basal level or CUEDC2-depleted condition (Supplementary Fig. S4a, b), and the cells being internalized post mitosis were negative in cleaved caspase3, a readout of apoptosis activation (Supplementary Fig. S4c-e).

internalized to form CIC structure (En). $n=12$. e Expression of p53 in inner cells of fresh CIC structures (CIC) is higher than that in single mono-nucleic cell (NC). $n=50$ for each group. $\mathbf{f}, \mathbf{g}$ p53 depletion (sip53) inhibits entotic CIC formation induced by CDC20 (siCD) and CUEDC2 (siCU) knockdown. siNC for nontarget control siRNA. h, $\mathbf{j}$ Reduced frequency for p53 knockdown (p53KD) cells (labeled with H2BmCherry) to penetrate into control cells (siNC). siNC for nontarget control siRNA.

\section{P53 is required for daughter cells to undergo entosis}

Since p53 is the key mediator of DNA damage signaling, we then explore the involvement of p53 in entosis. TLAS indicated that high level of p53 accumulated in the nuclei of daughter cells penetrating into their neighbors (Fig. 4a). The expression levels of p53 were positively correlated with $\gamma \mathrm{H} 2 \mathrm{AX}$ foci formation (Fig. 4b), and higher in mitotic cells of longer metaphase (Fig. 4c), and higher in the 

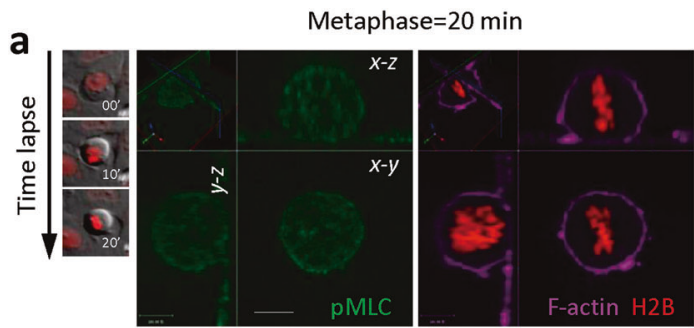

C
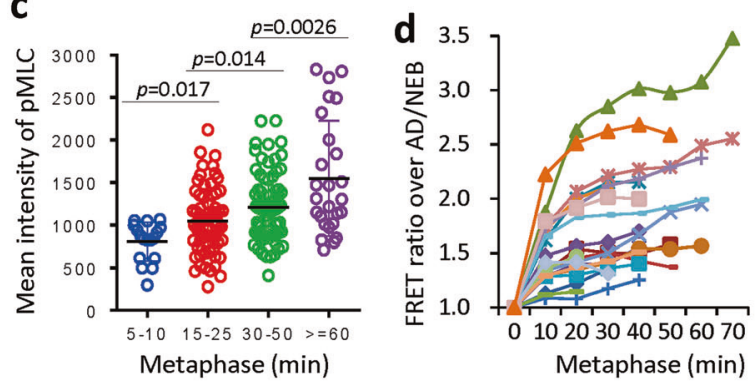

h
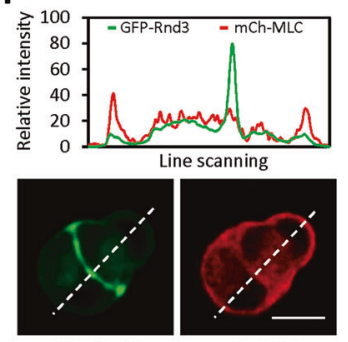

GFP-Rnd3

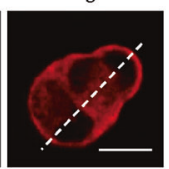

mCh-MLC

i

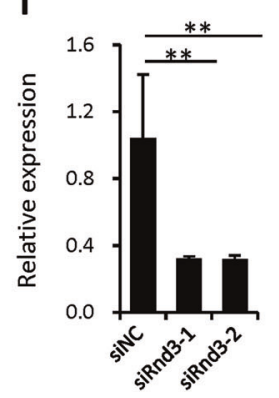

b

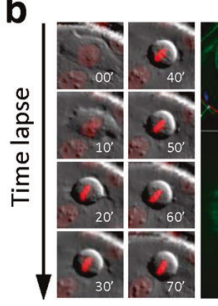

e

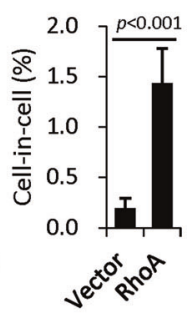

j

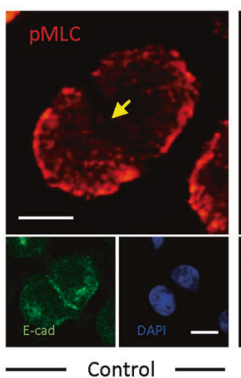

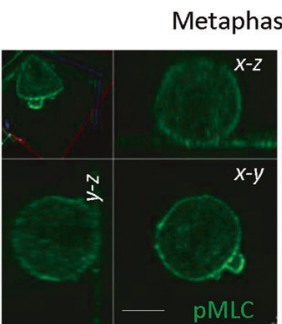

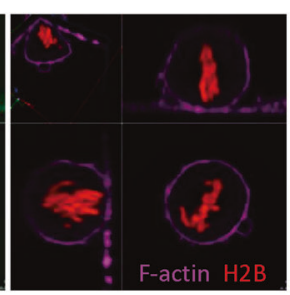

f

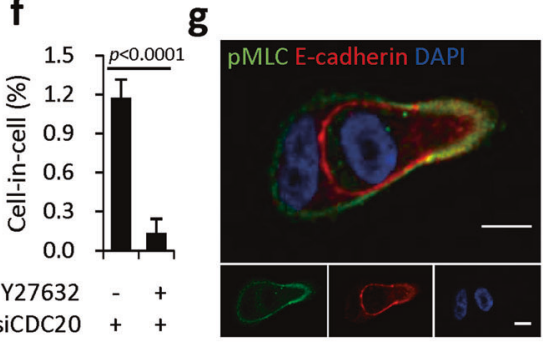

$\mathbf{k}$

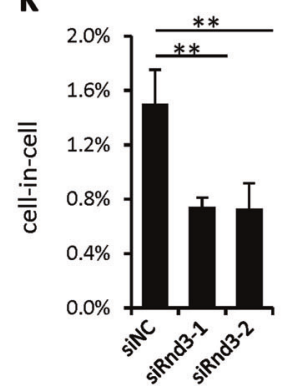

Fig. 5 Rnd3 is required for asymmetric RhoA activation and entotic CIC formation. a Representative image of pMLC staining in mitotic cell of normal metaphase $(20 \mathrm{~min})$, with corresponding DIC/mCherry images of time lapse on the left and F-actin staining on the right. Scale bar: $10 \mu \mathrm{m}$. Also see Movie S5. b Representative image of pMLC staining in mitotic cell of prolonged metaphase (60 min), with corresponding DIC/mCherry images of time lapse on the left and F-actin staining on the right. Scale bar: $10 \mu \mathrm{m}$. Also see Movie S5. c Graph plots pMLC mean intensity in mitotic cells of different metaphase duration as indicated. From left to right, $n=17$, $67,75,26$, respectively. Mean pMLC intensity of individual cell was calculated by normalization of background-subtracted pMLC intensity over cell area. Data are mean \pm SD of 20 fields with totally more than 10,000 of cells analyzed. The differences between groups are significant as shown by indicated $p$ values. d The dynamic changes of RhoA activity over metaphase duration measured by FRET analysis in 21 mitotic cells depleting CDC20. All background-subtracted mean FRET intensities were normalized over their corresponding mean FRET intensities at the time points of nuclear envelop breakdown (NEB) or cell adherence (AD) right before rounding up, whichever applied. Also see Movie S6. e Quantification of CIC structures in cells overexpressing RhoA. Data are mean \pm SD of 4 or more fields with more than 3000 cells analyzed each. $p<0.001$. f Inhibition of CIC formation in CDC20depleted cells by Y27632. Data are mean \pm SD of 4 or more fields with more than 5000 cells analyzed each. $p<0.0001$. g Polarized distribution of pMLC at the rear cortex of internalized cell in intermediate CIC structure. Scale bars: $10 \mu \mathrm{m}$. Also see Fig. S6e. h Rnd3-GFP localizes at the cell-cell junction during CIC formation. Upper graph shows line scan analysis, channeled images were shown underneath. Scale bar: $10 \mu \mathrm{m}$. i Rnd 3 mRNA level examined by qPCR upon knockdown by RNA interference. Data are mean \pm SD of triplicate experiment. $p<0.001$. j Representative images for junctional localization of pMLC2 (red) in MCF10A upon Rnd3 depletion (Rnd3 KD), E-cadherin (green) staining indicates cell junctions. Scale bars: $10 \mu \mathrm{m}$. Arrowheads indicate pMLC2 staining at cell junctions. Also see Fig. S6g. k Quantification of entotic CIC formation in control (siNC) and Rnd3 (siRnd3) depleted cells. Data are mean \pm SD of 4 or more fields with more than 5000 cells analyzed each. $p<0.01$. internalizing daughter cells as compared with their nonentotic sibling cells (Fig. 4d) and those from normal division (Fig. 4e). Importantly, RNA interference-mediated KD of p53 significantly suppressed entotic CIC formation induced by CDC20- or CUEDC2- KD (Fig. 4f, g), suggesting that p53 is required for entosis induced by prolonged mitosis. By a coculture experiment, p53 was demonstrated to function primarily in the mitotic daughter cells that penetrate into their neighbors, as p53 KD reduced the frequency of cells' penetration as inners when co-cultured with control cells (Fig. 4h-j). Despite the essential role of p53 in CIC formation, it seemed to have little impact on inner cell fates as the inner cell fate profiles are comparable between control and p53depleted groups (Supplementary Fig. S5). 

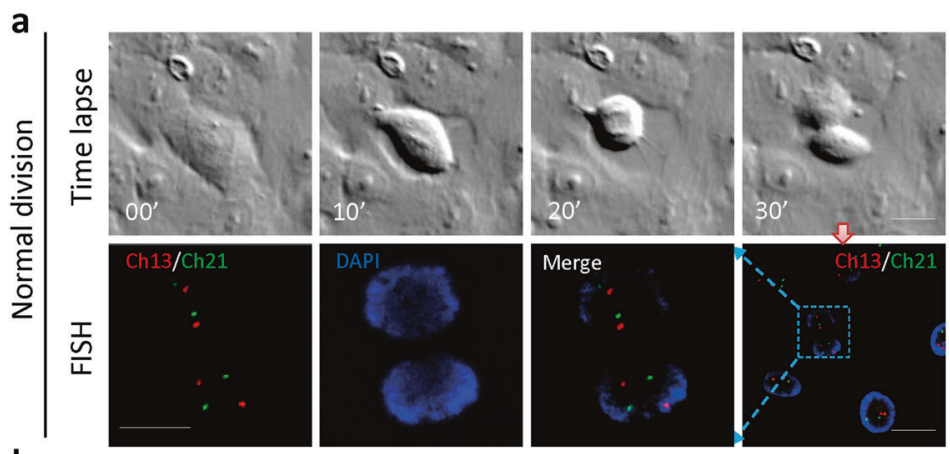

b
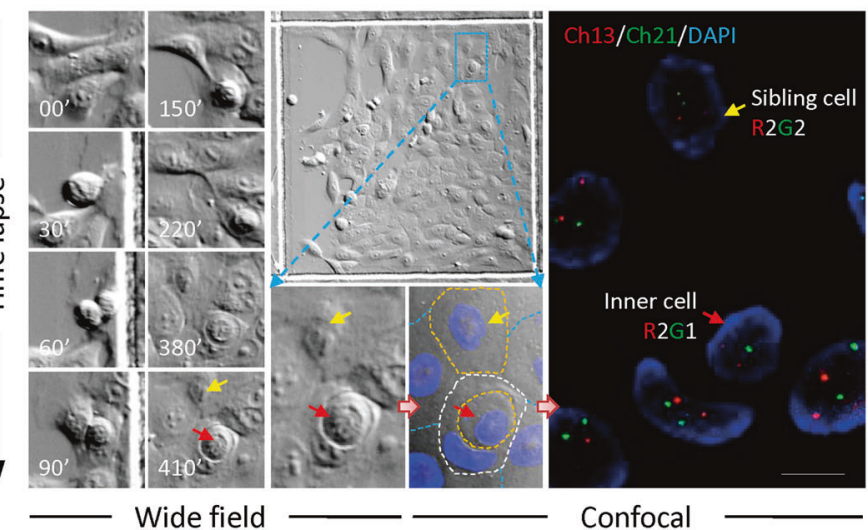

Confocal c

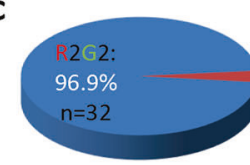

Single cells of normal division

e

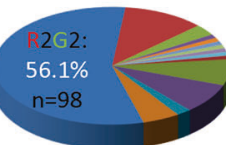

Inner cells of pre-existing $\mathrm{CIC}$

g

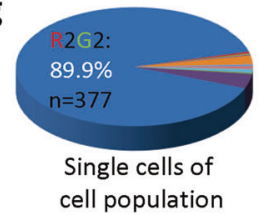

$\square$ R0G2 $\square$ R1G0 $\square$ R1G1 $\square \mathrm{R} 1 \mathrm{G} 2 \square \mathrm{R} 1 \mathrm{G} 3 \square \mathrm{R} 2 \mathrm{G} 0$ $\square \mathrm{R} 2 \mathrm{G} 2 \square \mathrm{R} 2 \mathrm{G} 1=\mathrm{R} 2 \mathrm{G} 3$ - R2G4 $\square$ R3G1 $-\mathrm{R} 3 \mathrm{G} 2$ - R3G3 $\square$ R3G4 $=\mathrm{R} 4 \mathrm{G} 2$ - R4G3 $=\mathrm{R} 4 \mathrm{G} 4=\mathrm{R} 5 \mathrm{G} 1$ - R6G3 R6G6 $\square$ R8G8 d

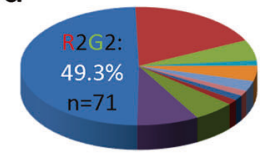

Inner cells of $\mathrm{ClC}$

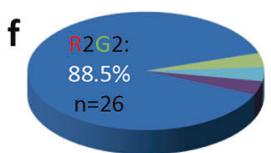

Sibling cells of $\mathrm{CIC}$

h

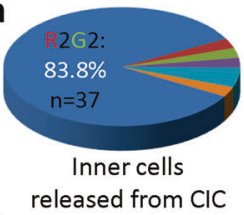

उ

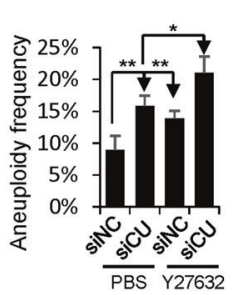

Fig. 6 Entosis selectively targets non-diploid cells for CICmediated death. a Representative images showing FISH result of normal cell division. Upper panel shows DIC image sequence of time lapse. Lower panel shows FISH results in two daughter cells. Scale bar: $20 \mu \mathrm{m}$ for right, $10 \mu \mathrm{m}$ for left. Also see Movie S7. b Representative images showing FISH result of cell division leading to CIC formation. Left panel shows DIC image sequence of time lapse. Middle panel shows the positional information of target cells/ structures in gridded glass bottom dish at the end of time lapse imaging. Right panel shows FISH results of selected region in middle panel. Scale bar: $10 \mu \mathrm{m}$. Yellow arrow indicates adherent sibling daughter cell, red arrow indicates daughter cell internalized to form CIC structure. FISH results are presented as $\mathrm{RnGn}, \mathrm{R}$ for red probe, $\mathrm{G}$ for green probe, $n$ for probe number. Also see Movie S7. Detail quantification and classification of FISH results for daughter cells of

\section{RhoA activity accumulated with prolonged metaphase promotes entosis}

To explore the potential mechanisms underlying the regulation of entosis by p53, we first examine changes in RhoA activities, which is prerequisite for CIC formation $[9,28]$ and regulated by p53 signaling [29, 30], during entosis. CDC20-depleted cells were stained with antibody for p-MLC, a readout of RhoA activities, following 4-h time lapse microscopy (Movie S5). As shown in Fig. 5a-c, mitotic cells of longer metaphase tended to display higher p-MLC intensity, in agreement with which, RhoA activities in mitotic cells did accumulate over time as monitored by FRET time lapse analysis (Fig. 5d, Supplementary Fig. S6a, b and Movie S6). Since entotic CIC formation occurred in daughter cells, we examined normal division with metaphase not more than $30 \mathrm{~min}$ (c), daughter cells from cell division that were internalized to form CIC structures (d), inner cells of pre-existing CIC structures of unknown origin (e), daughter cells from cell division that did not form CIC structures (f), single cells of unknown origin in the cell population (g), and internalized cells that were finally released from CIC structure (h), see Fig. S7b and Movie S7. FISH results are presented as RnGn, R for red probe, $\mathrm{G}$ for green probe, $n$ for probe number. The percentage of R2G2 together with cell number analyzed were shown for each pie picture. The related histories for all cells analyzed were determined by time lapse imaging. i Quantification of aneuploidy cells following entosis blockade. siNC nontarget control siRNA, siCU siRNA for CUEDC2. $* p<0.05$; $* *<<0.01$. For each condition, quantification was performed on $>400$ cells.

whether enhanced RhoA activities in mother cells could be inherited by daughter cells. RhoA activities of daughter cells right after cytokinesis were compared with those of their respective mother cells in the end of metaphase by FRET time lapse. As shown in Supplementary Fig. S6a-d, the two sibling daughter cells are tightly adherent to their mothers in RhoA activities, which is true for both normal and entotic cell division, suggesting that RhoA activities could be transmitted from mother cells to daughters with little loss. We then examined the effects of changed RhoA activities on entotic CIC formation, consistent with the essential role of RhoA in entosis, ectopic overexpression of RhoA significantly increased entotic CIC frequency (Fig. 5e), and inhibition of RhoA signaling by Y27632 targeting ROCKs efficiently suppressed entotic CIC formation (Fig. 5f), supporting a role of increased 


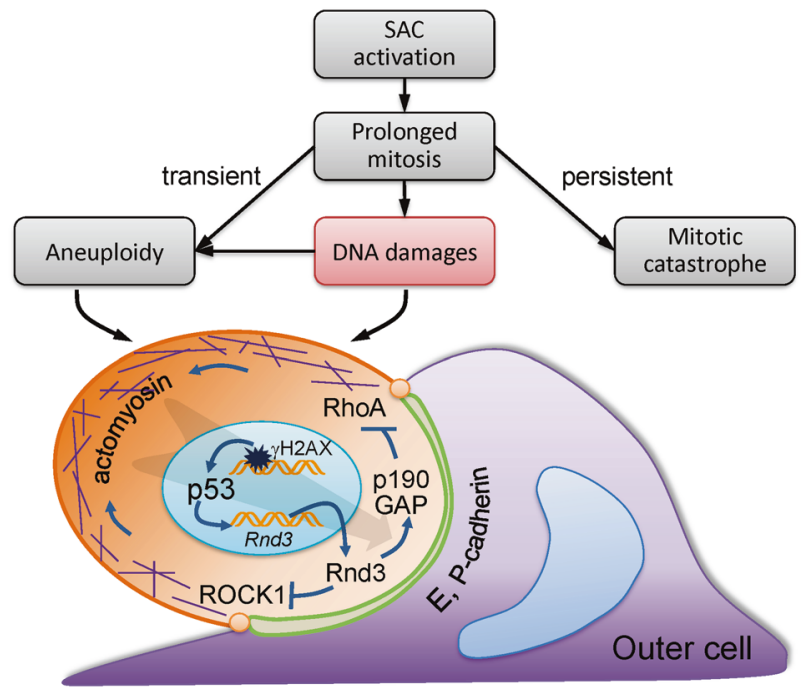

Fig. 7 Working model for p53-depedent postmitotic surveillance by entosis. SAC activation leads to mitotic arrest characterized by prolonged metaphase. While persistent mitotic arrest activates cell death by mitotic catastrophe prior to cytokinesis, transient mitotic arrest may cause DNA damages that promote chromosome missegregation to give rise to daughter cells of aneuploidy. DNA damages marked by $\gamma \mathrm{H} 2 \mathrm{AX}$ activate DDR signaling and p53 in daughter cells. p53 upregulates the expression of downstream target Rnd3 which, by targeting ROCK1 and p190 RhoGAP, inhibits RhoA activities at cell-cell junction where lie E-, P-cadherin, leading to polarized activation of RhoA-actomyosin at rear cortex which drives cell internalization to form CIC structures. Subsequent death promoted by the outer cell eliminates those aneuploid daughter cells to maintain genome integrity.

RhoA activity in promoting entosis induced by prolonged mitosis.

\section{P53-regulated Rnd3 is essential for compartmentalizing RhoA activities and entosis}

Since polarized distribution of actomyosin, the downstream effector of RhoA signaling, is essential for cells' penetration into their neighboring cells [9], we examined the expression of p-MLC, the readout of contractile actomyosin and RhoA activity, in the intermediate entotic CIC structures. As shown in Fig. 5g and Supplementary Fig. 6e, p-MLC displays typical asymmetric distribution pattern, characterized by higher intensity at the rear cortex than that at cell-cell junctions marked by E-cadherin where RhoA activities were actively suppressed by inhibitors such as p190A RhoGAP [9]. While wild-type p53 was known to negatively regulate RhoA signaling through its downstream target Rnd3 [31, 32], an atypical Rho GTPase that inhibits RhoA signaling by directly targeting ROCK I [31, 33] and p190A RhoGAP [34]. We therefore hypothesized that Rnd3 may facilitate CIC formation via helping compartmentalize RhoA activities. In agreement with this notion, Rnd3, regulated by p53 (Supplementary Fig. S6f), specifically localizes at cell-cell junctions while MLC is enriched at the cortical periphery away from junctions during CIC formation (Fig. 5h). SiRNA-mediated KD of Rnd3 led to increased pMLC staining at cell-cell junctions (Fig. 5i, j and Supplementary Fig. S6g), suggesting that the junctional-localization of Rnd3 spatially restricts RhoA pathway activity and inhibits myosin contraction at cell-cell junctions, which promotes CIC formation. Consequently, Rnd3 depletion significantly inhibited entotic CIC formation (Fig. 5k). Together, the above data fits a model whereby p53-regulated Rnd3 compartmentalizes RhoA activity in daughter cells from prolonged mitosis to drive CIC formation.

\section{Entosis selectively targets mitotic progenies of non- diploidy}

Prolonged metaphase due to SAC activation allows cells to fix problems during chromosome segregation. Nevertheless, prolonged mitosis also elicit structural DNA damages [27, 35] that, via truncated DDR, induce wholechromosome missegregation [36], leading to non-diploid daughter cells [37]. We therefore hypothesized that entosis was set to ensure selective elimination of those non-diploid cells. Fluorescent in situ hybridization (FISH) was employed to access ploidy changes in MCF10A cells plated on gridded glass bottom dish following $24 \mathrm{~h}$ time lapse microscopy, and the metaphase and position histories of target cells were determined by retroactive review of the time lapse records (Movie S7). While mitosis of short metaphase, based on the probes used, generally gave rise to diploid progenies (96.9\%) (Fig. 6a, c), cells of prolonged metaphase penetrating their neighbors to form CIC structures were largely non-diploid (50.7\%) (Fig. 6b, d and Supplementary Fig. S7a). This non-diploid rate of entotic inner cells is similar to that (43.9\%) of inner cells of pre-existing CIC structures (Fig. 6e), consistent with our observation that majority of CIC structures were from prolonged mitosis. Intriguingly, the sibling cells that did not participate into CIC formation were largely diploid (88.5\%) (Fig. 6f) in a rate similar to the whole MCF10A population (89.9\%) (Fig. 6g). And a small portion of inner cells that eventually got released from CIC structures were also largely diploid (83.8\%) (Fig. 6h and Supplementary Fig. S7b). Therefore, entosis selectively targets non-diploid daughter cells for elimination, which may play an essential role in maintaining population genetic integrity, as blocking entosis by Y27632, significantly increased non-diploid cells in the cell population (Fig. 6i). Thus, our data support that p53regulated entosis may serve as a cellular mechanism of mitotic surveillance. 


\section{Discussion}

In summary, we proposed a CIC-mediated mechanism of postmitotic surveillance as entosis whereby non-diploid progenies from prolonged mitosis were eliminated in a p53-dependent manner. In this model (Fig. 7), $\gamma \mathrm{H} 2 \mathrm{AX}-$ marked DNA damages associated with prolonged mitosis in mother cells activate p53 pathway in daughter cells that are non-diploid due to chromosome missegregation, p53 upregulates the expression of its downstream target Rnd3 which locally inhibits RhoA signaling and actomyosin contraction at cell-cell junctions, leading to asymmetric RhoA activation at the rear cortex of daughter cells to drive cell internalization and subsequent non-apoptotic death. Thus, this work uncovered a novel non-apoptotic mechanism by entosis for p53 to maintain genome integrity. This mechanism turns out to work not only in MCF10A cells, but also in HMECs, another line of non-transformed epithelial cells (Supplementary Fig. S8), and also in MCF7 cells, a cancer cell line derived from breast carcinoma (Supplementary Fig. S9), suggesting that it might be a mechanism functional in multiple cellular contexts.

DNA damages associated with mitosis are generally phenotyped as prolonged mitosis or mitotic arrest, which could also cause DNA damages [27, 35]. Formation of this vicious loop is largely due to impaired DDR which, though being capable of sensing DNA damages, likely stops short of downstream damage repair pathways [38]. Though activation of this partial DDR was believed to prevent fusion of exposed telomeres under normal circumstance [39], it left unrepaired DNA damages, pre-mitotic or acquired, into mitotic progenies under stressed conditions [40]. Moreover, activation of the partial DDR during mitosis, instead helps fix errors in DNA, actually could result in severe chromosome missegregation, which eventually gives rise to aneuploid progenies [36, 37]. Therefore, surveillance mechanisms are critical to deal with daughter cells from prolonged mitosis. Previous studies indicated that prolonged mitotic progenies might be surveilled by either growth arrest or apoptosis [5, 21, 41]. Whereas in this study, we identified a novel surveillance mechanism executed by CIC-mediated non-apoptotic death termed entosis. Although also being activated by prolonged mitosis via p53-dependent pathway, entosis likely represents a unique process independent from growth arrest and apoptosis because depleting p21, a p53 downstream effector that is required for growth arrest/senescence, did not block entotic CIC formation at all (data not shown), and depletion of Rnd3, a p53 target gene that was identified as suppressor of ROCK I-mediated apoptosis [31, 42], significantly suppress entosis (Fig. 5j, k).

Interestingly, while our work demonstrates a positive role of CIC formation in maintaining genome stability by eliminating aneuploidy cells, previous work supported entosis as a cellular mechanism to promote genome instability by inducing aneuploidy [18]. This clear conceptual discrepancy is actually due to different cellular and molecular contexts where CIC formation may work. At cellular level, Krajcovic et al. found that the presence of inner cell could physically block cytokinesis of outer cell, leading to outer cell binucleation and subsequent aneuploidy of its offspring following next round of cell division [18]. This effect may be propagated to promote genome instability in the context of tumor cells that are tolerated to aneuploidy. This idea was supported by recent work in lung cancer cells Mackay et al. [19], whereas our work investigates CIC's role in a non-tumor context, in which aneuploid daughters from prolonged mitosis were internalized as inner cells and eliminated. Thus, it is likely that CIC formation affects genome stability differentially via inner cell and outer cell, respectively, depending on cells' tolerance to aneuploidy. For tumor cells who are generally aneuploidy-tolerant, CIC formation may promote genome instability by inducing cytokinesis failure of outer cells; for non-transformed epithelial cells, CIC formation may function to counteract genome instability by internalizing and eliminating aneuploid cells. Of note, since aneuploidy is toxic and lethal to non-transformed cells, the aneuploid progenies of binucleated outer cells would not survive for long period. This is also true for tumor cells that are not tolerant to aneuploidy [19]. Therefore, CIC formation is genome instability- or tumorsuppressive anyway when cells are not tolerant to aneuploidy. At molecular level, CIC's effects on aneuploidy and genome stability are controlled by p53 status. While Mackay et al. found that only mutant p53, but not wildtype or null p53, could promote genome instability via CIC formation by conferring cancer cells winner/outer identity and allowing survival and population of their aneuploidy progenies [19], our work demonstrated that wild-type p53 endowed loser/inner identity to cells during CIC formation by facilitating the establishment of polarized RhoA activities. Together, we propose that CIC formation, depending on p53 status, may play dual roles in genome instability and tumorigenesis as well. For cancer cells with mutant p53, CIC formation plays promotive role by facilitating selection of cancer cells harboring mutant p53; for normal epithelial cells or cancer cells that contain wild-type p53, CIC formation plays suppressive role by eliminating aneuploidy cells expressing wild-type p53 through entosis. It will be of interest to explore whether other oncogenic mutations may also regulate CIC's functional outcomes.

Recently, two other works reported entosis induction in confined contexts in adherent monolayer culture [26, 43]. One work demonstrated that glucose starvation, an 
extreme biological condition, was capable of promoting CIC formation mainly in the context of cancer cells like MCF7 in an AMP activated protein kinase (AMPK)dependent way [43]. Interestingly, although AMPK is a well-known energy sensor, it was found in mitotic apparatus and involved in the regulation of mitotic progression and completion [44, 45]. We hypothesize that glucose induced activation of AMPK may influence normal mitosis, which triggers entosis and contributes to CIC formation in adherent cells that is yet to be validated. Another work reported that the depletion of $\mathrm{CDC} 42$, a polarity protein, induced CIC formation in 16HBE cells [26]. Although mitosis was found linked to CIC formation, the work failed to identify the essential role of mitotic arrest and DNA damages in CIC formation, hence, could not tell why most mitosis failed to give rise to CIC structures and why reagents blocking mitosis unexpectedly induced CIC formation. Similar to work by Wan et al. [46], where depletion of polarity protein PAR3 resulted in enhanced formation of CIC structures in adherent MDCK cells, polarity changes were not regarded as the reason for enhanced CIC formation [26, 46]. Actually, we found that depleting polarity protein such as CDC42 in MCF10A and tumor cells could promote CIC formation that was also preceded by prolonged mitosis (data not shown), indicated us that altered mitosis might be a shared route for multiple factors, such as glucose starvation and depleting polarity proteins and the like, to activate entosis that works in multiple contexts to maintain homeostasis.

Acknowledgements We thank Dr. Overholtzer from Memorial SloanKettering Cancer Center and Dr. Qinong Ye from Beijing Institute of Biotechnology for providing cell lines and reagents. We thank Dr. Louis Hodgson from Albert Einstein College of Medicine of Yeshiva University for assistance in FRET. We thank Dr. Dangsheng Li for discussions and critical reading of the manuscript. This work was supported by the National Key Research \& Development Program of China (2016YFC1303303, 2019YFA09003801, 2018YFA0900804), the National Natural Science Foundation of China (31970685, $31671432,81872314,31770975,81572799)$. QS conceived the project and conception. QS, HH, and JL designed experiments with the advice from XNW and ZC. JL performed majority of the experiments with the assistance of $\mathrm{XY}$ for RNA interference, phenotype, and qPCR, and ZN for pathology, immunostaining, and FISH. MW, BZ, and HQ helped in protein expression and detection. SG and LG assisted imaging. YT provided patient samples and related information. QS, HH, and JL analyzed the data with the assistance of $\mathrm{YZ}, \mathrm{CW}$, HR, BR, XZ, XS. QS, and JL wrote the paper with input from XNW, $\mathrm{LM}$, and $\mathrm{HH}$, and all authors reviewed the manuscript.

\section{Compliance with ethical standards}

Conflict of interest The authors declare that they have no conflict of interest.

Publisher's note Springer Nature remains neutral with regard to jurisdictional claims in published maps and institutional affiliations.

\section{References}

1. Sivakumar S, Gorbsky GJ. Spatiotemporal regulation of the anaphase-promoting complex in mitosis. Nat Rev Mol Cell Biol. 2015;16:82-94.

2. Musacchio A. The molecular biology of spindle assembly checkpoint signaling dynamics. Curr Biol. 2015;25:R1002-18.

3. Kapanidou M, Curtis NL, Bolanos-Garcia VM. Cdc20: at the crossroads between chromosome segregation and mitotic exit. Trends Biochem Sci. 2017;42:193-205.

4. Gao YF, Li T, Chang Y, Wang YB, Zhang WN, Li WH, et al. Cdk1-phosphorylated CUEDC2 promotes spindle checkpoint inactivation and chromosomal instability. Nat Cell Biol. 2011; 13:924-33.

5. Vitale I, Galluzzi L, Castedo M, Kroemer G. Mitotic catastrophe: a mechanism for avoiding genomic instability. Nat Rev Mol Cell Biol. 2011;12:385-92.

6. Santaguida S, Amon A. Short- and long-term effects of chromosome mis-segregation and aneuploidy. Nat Rev Mol Cell Biol. 2015;16:473-85.

7. Funk LC, Zasadil LM, Weaver BA. Living in CIN: mitotic infidelity and its consequences for tumor promotion and suppression. Dev Cell. 2016;39:638-52.

8. Overholtzer M, Mailleux AA, Mouneimne G, Normand G, Schnitt SJ, King RW, et al. A nonapoptotic cell death process, entosis, that occurs by cell-in-cell invasion. Cell. 2007;131:966-79.

9. Sun Q, Cibas ES, Huang H, Hodgson L, Overholtzer M. Induction of entosis by epithelial cadherin expression. Cell Res. 2014;24:1288-98.

10. Wang M, Niu Z, Qin H, Ruan B, Zheng Y, Ning X, et al. Mechanical ring interfaces between adherens junction and contractile actomyosin to coordinate entotic cell-in-cell formation. Cell Rep. 2020;32:108071.

11. Liang J, Fan J, Wang M, Niu Z, Zhang Z, Yuan L, et al. CDKN2A inhibits formation of homotypic cell-in-cell structures. Oncogenesis. 2018;7:1-8.

12. Wang C, Chen A, Ruan B, Niu Z, Su Y, Qin H, et al. PCDH7 inhibits the formation of homotypic cell-in-cell structure. Front Cell Dev Biol. 2020;8:1-12.

13. Ruan B, Wang C, Chen A, Liang J, Niu Z, Zheng Y, et al. Expression profiling identified IL-8 as a regulator of homotypic cell-in-cell formation. BMB Rep. 2018;51:412-7.

14. Ruan B, Zhang B, Chen A, Yuan L, Liang J, Wang M, et al. Cholesterol inhibits entotic cell-in-cell formation and actomyosin contraction. Biochem Biophys Res Commun. 2018;495:1440-6.

15. Florey O, Kim SE, Sandoval CP, Haynes CM, Overholtzer M. Autophagy machinery mediates macroendocytic processing and entotic cell death by targeting single membranes. Nat Cell Biol. 2011;13:1335-43.

16. Sun Q, Luo T, Ren Y, Florey O, Shirasawa S, Sasazuki T, et al. Competition between human cells by entosis. Cell Res. 2014;24: 1299-310.

17. Kroemer G, Perfettini J-L. Entosis, a key player in cancer cell competition. Cell Res. 2014;24:1280-1.

18. Krajcovic M, Johnson NB, Sun Q, Normand G, Hoover N, Yao E, et al. A non-genetic route to aneuploidy in human cancers. Nat Cell Biol. 2011;13:324-30.

19. Mackay HL, Moore D, Hall C, Birkbak NJ, Jamal-Hanjani M, Karim SA, et al. Genomic instability in mutant p53 cancer cells upon entotic engulfment. Nat Commun. 2018;9:3070.

20. Bieging KT, Mello SS, Attardi LD. Unravelling mechanisms of p53mediated tumour suppression. Nat Rev Cancer. 2014;14:359-70.

21. Joerger AC, Fersht AR. The p53 pathway: origins, inactivation in cancer, and emerging therapeutic approaches. Annu Rev Biochem. 2016;85:375-404. 
22. Kruiswijk F, Labuschagne CF, Vousden KH. p53 in survival, death and metabolic health: a lifeguard with a licence to kill. Nat Rev Mol Cell Biol. 2015;16:393-405.

23. Ranjan A, Iwakuma T. Non-canonical cell death induced by p53. Int J Mol Sci. 2016;17:2068.

24. Sun Q, Overholtzer M. Methods for the study of entosis. In: McCall K, Klein C, editors. Methods in Mol Biol-Necrosis, Vol. 1004. Totowa, NJ: Humana Press; 2013, pp 59-66.

25. Pertz O, Hodgson L, Klemke RL, Hahn KM. Spatiotemporal dynamics of RhoA activity in migrating cells. Nature. 2006; 440:1069-72.

26. Durgan J, Tseng YY, Hamann JC, Domart MC, Collinson L, Hall A, et al. Mitosis can drive cell cannibalism through entosis. eLife. 2017;6:1-26.

27. Ganem NJ, Pellman D. Linking abnormal mitosis to the acquisition of DNA damage. J Cell Biol. 2012;199:871-81.

28. Ning X, Luo T, Chen Z, Sun Q. The physics for the formation of cell-in-cell structures. Curr Mol Med. 2015;15:867-72.

29. Xia M, Land H. Tumor suppressor p53 restricts Ras stimulation of RhoA and cancer cell motility. Nat Struct Mol Biol. 2007;14:215-23.

30. Gadea G, de Toledo M, Anguille C, Roux P. Loss of p53 promotes RhoA-ROCK-dependent cell migration and invasion in 3D matrices. J CeLL Biol. 2007;178:23-30.

31. Ongusaha PP, Kim HG, Boswell SA, Ridley AJ, Der CJ, Dotto GP, et al. RhoE is a pro-survival p53 target gene that inhibits ROCK I-mediated apoptosis in response to genotoxic stress. Curr Biol. 2006;16:2466-72.

32. Zhu Y, Zhou J, Xia H, Chen X, Qiu M, Huang J, et al. The Rho GTPase RhoE is a p53-regulated candidate tumor suppressor in cancer cells. Int J Oncol. 2014;44:896-904.

33. Riento K, Guasch RM, Garg R, Jin B, Ridley AJ. RhoE binds to ROCK I and inhibits downstream signaling. Mol Cell Biol. 2003; 23:4219-29.

34. Wennerberg K, Forget M-A, Ellerbroek SM, Arthur WT, Burridge $\mathrm{K}$, Settleman J, et al. Rnd proteins function as RhoA antagonists by activating p190 RhoGAP. Curr Biol. 2003;13:1106-15.
35. Dalton WB, Nandan MO, Moore RT, Yang VW. Human cancer cells commonly acquire dna damage during mitotic arrest. Cancer Res. 2007;67:11487-92.

36. Bakhoum SF, Kabeche L, Murnane JP, Zaki BI, Compton DA. DNA-damage response during mitosis induces wholechromosome missegregation. Cancer Discov. 2014;4:1281-9.

37. Bakhoum SF, Kabeche L, Compton DA, Powell SN, Bastians H. Mitotic DNA damage response: at the crossroads of structural and numerical cancer chromosome instabilities. Trends Cancer. 2017; 3:225-34.

38. Heijink AM, Krajewska M, van Vugt MA. The DNA damage response during mitosis. Mutat Res. 2013;750:45-55.

39. Orthwein A, Fradet-Turcotte A, Noordermeer SM, Canny MD, Brun CM, Strecker J, et al. Mitosis inhibits DNA double-strand break repair to guard against telomere fusions. Science. 2014;344: 189-93.

40. Denchi EL, Li J. Let it go: how to deal with a breakup in mitosis. Nat Struct Mol Biol. 2014;21:433-5.

41. Lambrus BG, Holland AJ. A new mode of mitotic surveillance. Trends Cell Biol. 2017;27:314-21.

42. Paysan L, Piquet L, Saltel F, Moreau V. Rnd3 in cancer: a review of the evidence for tumor promoter or suppressor. Mol Cancer Res. 2016;14:1033-44.

43. Hamann JC, Surcel A, Chen R, Teragawa C, Albeck JG, Robinson $\mathrm{DN}$, et al. Entosis is induced by glucose starvation. Cell Rep. 2017;20:201-10.

44. Banko MR, Allen JJ, Schaffer BE, Wilker EW, Tsou P, White JL, et al. Chemical genetic screen for AMPKalpha2 substrates uncovers a network of proteins involved in mitosis. Mol Cell. 2011;44:878-92.

45. Li Z, Zhang X. Kinases involved in both autophagy and mitosis. Int J Mol Sci. 2017;18:1884.

46. Wan Q, Liu J, Zheng Z, Zhu H, Chu X, Dong Z, et al. Regulation of myosin activation during cell-cell contact formation by Par3Lgl antagonism: entosis without matrix detachment. Mol Biol Cell. 2012;23:2076-91. 